\title{
Near-Field Beamforming at Intelligent Re-Configurable Surface in Uplink Transmission
}

\author{
Visa Tapio, Markku Juntti \\ Centre for Wireless Communications (CWC) \\ Uninvesity of Oulu \\ Oulu, Finland \\ e-mail: FirsName.LastName@oulu.fi
}

\author{
Ibrahim Hemadeh, Alain Mourad \\ InterDigital Europe, Ltd \\ London, United Kingdom \\ e-mail: FirstName.LastName@InterDigital.com
}

\begin{abstract}
Intelligent re-configurable surfaces (IRS) constitute electronically steerable structures that can be used to control the propagation of electromagnetic (EM) waves. Owing to their passive nature in terms of signal reflection, IRS require largescale implementations. This means that the near-field effect, e.g., the curvature of the propagating EM wave must be considered. In this paper, a simple two-step near-field beamforming method is proposed. In the first step, the direction from the IRS center point to the user equipment (UE) is found assuming plane wave model. In the second step, the transmitted beam is focused towards the UE based on the pre-calculated constant phase contours. All operations in the proposed method can be implemented as memory operations, i.e., no complex computations are needed. The operation of the method is verified with simulations. The results show that the proposed beamforming technique is capable of focusing the beam pattern of the IRS towards the user. More specifically, the near-field focusing of a $(50 \times 50)$-element IRS operating at $5 \mathrm{GHz}$ frequency increases the received power by $3 \mathrm{~dB}$ when compared to a case where the near-field effect is ignored.
\end{abstract}

Index Terms-Intelligent re-configurable surface, beam steering, near-field

\section{INTRODUCTION}

INTELLIGENT radio surface (IRS) is a programmable structure that can be used for controlling the propagation of electromagnetic (EM) waves by changing the electric and magnetic properties of the surface. In addition to the control of EM waves, IRSs can be used to sense the radio environment by integrating sensing capabilities into them. By placing intelligent surfaces into the environment where wireless systems are operating, the properties of the radio channels can be partially controlled [1], [2], [3].

In an IRS assisted wireless system, the transmitted signal is reflected by the IRS and the reflected signal is received at a receiver. In order to maximize the signal power at the receiver, the reflected energy must be directed to the right direction. The amount of energy the IRS can reflect depends on its size. Since the IRS does not amplify the reflected signals but rather passively steer them, its required size can be large. This means that the receiver can be in the near-field of the IRS. In this case, the effect of the curvature of the propagating EM wave must be considered in order to maximize the received energy at the receiver.
Near-field effect is considered in [4], where a uniform linear array with 64 elements is used to localize UEs in an indoor environment. Some of the UEs are in the near-field of the array while others are in the far field. The processing in [4] is based on the sparse Bayesian reconstruction method. In [5], the near-field effect is considered in an imaging radar application. The direction and distance of the targets in [5] are found by dividing the large array into smaller arrays that do not suffer from the near-field effect. After the direction and distance have been found, they are used in the compensation of the near-field effect. A convex optimization based near-field beamformer for a uniform linear array is described in [6]. A near-field beamformer using successive cancellation scheme for a uniform circular array is reported in [7]. The above near-field beamformers have been developed for multi-antenna transceivers. In [8], it has been shown from an EM perspective that a reflecting surface consisting of a sub-wavelength units can be used for near-field beamforming.

In multiple-input multiple output (MIMO) and radar systems, the signals at each antenna element, or at least a subset of the elements, are available for measurement in the receiver. In IRS-based beamforming, where all the elements are passive in the sense that there are no transceivers connected to them, the signals at IRS elements are not available for processing. Hence, all the processing is based on the received signal at a receiver distant from the IRS. The beamforming method proposed in this paper is based on simple search procedures. The direction from the IRS to the AP is assumed to be known. Since both the AP and IRS are typically installed at fixed positions, this assumption is generally valid. It is also assumed that the properties of the IRS are known, particularly the phase distribution of the received signal, when the UE is directly in front of the IRS center point at different distances are known. This information can be measured and stored during the manufacturing of the IRS. Based on the known characteristics of the IRS, the phase values required at the IRS side can be estimated with the stored phase values and power measurements at the receiver without any complex mathematical operations. The operation and performance of the proposed method is studied with simulations. The geometry of the array in simulations is assumed to be a planar square array, but the same method can be used with any array geometry. 
The rest of the paper is organized as follows. The system model is described in Section II. The proposed near-field beamforming method is presented in Section III and numerical examples are given in Section IV, Finally, conclusions are discussed in Section V.

\section{System Model}

Consider the system depicted in Fig. 1. More specifically, this system consists of an access point (AP), a single antenna $\mathrm{UE}$ and an IRS. The IRS is comprised of $(N \times M)$ elements with uniform spacing placed on the $x-y$ plane planar array, while the AP is equipped with at least one antenna element. The antenna elements at the AP side are arranged in a linear array with element spacing $\lambda / 2$, with $\lambda$ being the signal wavelength, given that the number of antenna elements in the AP is more than one. In this scenario, the signal transmitted by the UE reaches the AP via the IRS of Fig. 1. It is assumed that there is no direct link between the UE and the AP. The variables $x, y$ and $z$ in Fig. 1 are the horizontal and vertical distances from the IRS center, and the distance from the wall on which the IRS is attached, respectively.

It is assumed that the distance between the UE and the IRS is short in the sense that the signal received by the IRS cannot be assumed to be a plane wave, i.e., the curvature of the received EM must be considered. Hence, given that the UE in Fig. 1 transmits a signal $s(t)$, the signal impinged at the $(n, m)$-element of the IRS can be expressed as

$$
s_{\mathrm{IRS}}(n, m)=\alpha_{n, m} e^{-j k\left|\mathbf{r}_{n, m}\right| / c} s(t),
$$

where $k=2 \pi / \lambda, c$ is the speed of light, $\mathbf{r}_{n, m}$ denotes the vector from the UE antenna to the element $(n, m)$ and $\alpha_{n, m}$ is the path loss between the antenna and the IRS element, respectively. Furthermore, the signal reflected by the $(n, m)$ element is

$$
s_{\mathrm{IRS}, \mathrm{r}}(n, m)=\left|g_{n, m}\right| e^{j \phi_{n, m}} s_{\mathrm{IRS}}(n, m),
$$

where $g_{n, m}$ is the reflection coefficient of the element $(n, m)$ having the phase $\phi_{n, m}$. Here, the IRS can be controlled by tuning the phase $\phi_{n, m}$.

The distance between the IRS and AP is assumed to be long enough to allow the plane wave assumption. Further, it is assumed that there is only one path between the IRS and BS and the direction of IRS from the AP is known. No other information is needed. Here, the signal vector received by the AP antenna array is

$$
\mathbf{s}_{\mathrm{AP}}=\mathbf{s}(\varphi) h \sum_{n=1}^{N} \sum_{m=1}^{M} s_{\mathrm{IRS}, \mathrm{r}}(n, m),
$$

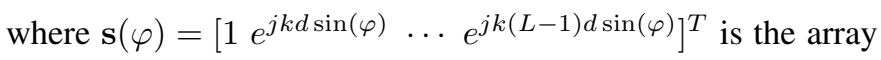
response vector of the signal arriving from angle $\varphi$ to the BS antenna array and $h$ is the channel gain between the IRS and AP. In the following section, we present our proposed IRSbased near-field BF technique.

\section{NEAR-FIELD BEAMFORMING AT IRS}

When an EM wave arrives at the IRS from a nearby UE, the phase distribution of the arriving signal varies depending on the direction and distance between the UE and IRS. An example of the phase distribution on the IRS is illustrated in Fig. 2a. The curvature of the constant phase contours is due to the near-field effect. The phase pattern in Fig. 2b is from the case when the UE is directly in front of the IRS $(x=0, y=0$ in Fig. 1). In this case the constant phase contours form co-centric circles on the IRS. Now, when the size of the IRS and the frequency of the EM wave are known, the phase pattern induced by the UE can be calculated offline and saved to the memory of the IRS controller. Hence, the near-field beamforming can be applied using a simple search operation. In general, the elevation and azimuth angles between the UE position and the IRS should be estimated prior to applying the near-field BF. After the angles have been estimated, the coordinate systems can be rotated so that the phase distribution changes from the general case into the co-centric circles. Hence, the beamforming can be done in two steps: 1) find the azimuth and elevation angles assuming plane wave model, 2) focus the beam with a search algorithm.

Given that the AP and IRS positions are assumed to be fixed, the direction from the IRS to the AP is assumed to be known, i.e., the required angle of reflection from the IRS is known. The problem is then to find the correct phase values on the IRS so that the signal transmitted by the UE is reflected towards the correct direction. The two-steps of the proposed near-field BF technique are summarized as follows:

Step 1: When the impinging wave on the IRS is assumed to be a plane wave, the relative phase of the wave at the $(n, m)$ element is [9]

$$
\psi_{i}(n, m)=\kappa d\left[\left(n \sin \left(\theta_{i}\right) \cos \left(\phi_{i}\right)+m \sin \left(\theta_{i}\right) \sin \left(\phi_{i}\right)\right]\right.
$$

where $-M / 2 \leq m \leq M / 2,-N / 2 \leq N \leq N / 2$ ( $N, M$ even), $\kappa=2 \pi / \lambda, d$ is the element spacing in horizontal and vertical directions, $\theta_{i}$ is the angle between the IRS normal vector and the direction of wave propagation and $\phi_{i}$ is the angle of the wave propagation projected on the IRS surface.

If the signal is to be reflected to direction $\left(\theta_{r}, \phi_{r}\right)$, the phase of the signal reflected by the element $(n, m)$ must be

$$
\psi_{r}(n, m)=\kappa d\left[\left(n \sin \left(\theta_{r}\right) \cos \left(\phi_{r}\right)+m \sin \left(\theta_{r}\right) \sin \left(\phi_{r}\right)\right] .\right.
$$

The required phase shift at the element is then

$$
\begin{array}{r}
\Delta(n, m)=\kappa d\left[n\left(\sin \left(\theta_{r}\right) \cos \left(\phi_{r}\right)-\sin \left(\theta_{i}\right) \cos \left(\phi_{i}\right)\right)+\right. \\
\left.m\left(\sin \left(\theta_{r}\right) \sin \left(\phi_{r}\right)-\sin \left(\theta_{i}\right) \sin \left(\phi_{i}\right)\right)\right]
\end{array}
$$

To find the direction angles $\theta_{i}, \phi_{i}$, the AP measures the received signal power while the search space is scanned. The phase values giving the maximum received power are selected as the angle estimates. After the direction angles have been found, the phase shifts required on the IRS surface are calculated with (6).

Step 2: The mechanical equivalent of Step 1 would be turning the IRS towards the UE. In the mechanical equivalent 


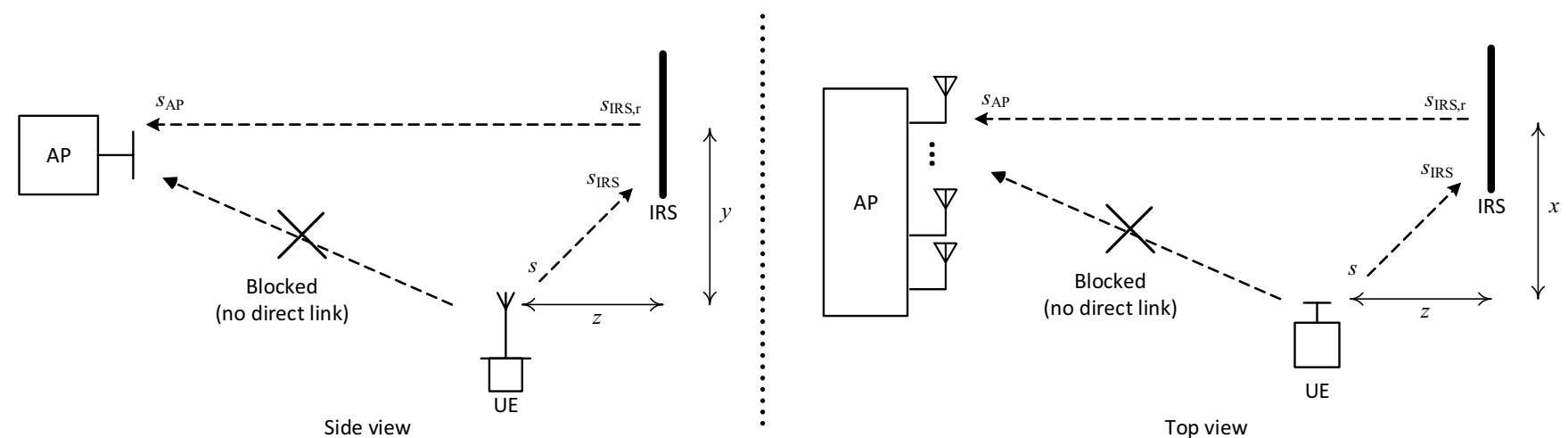

Fig. 1: Indoor scenario

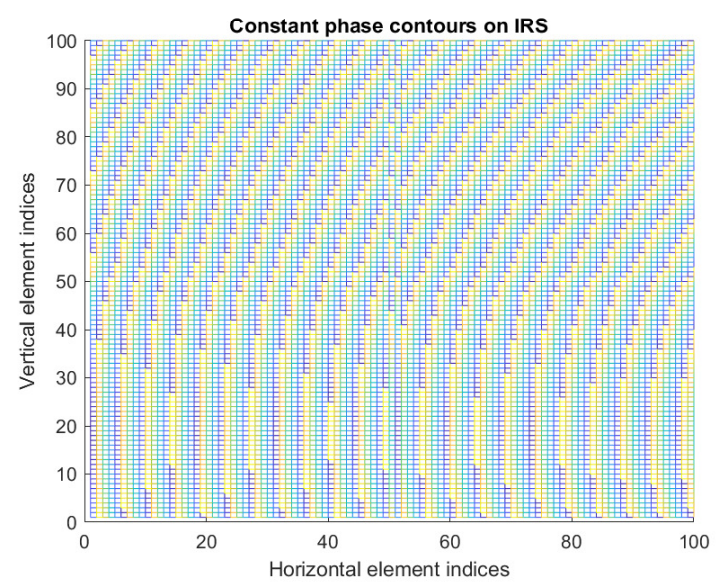

(a) Unknown direction.

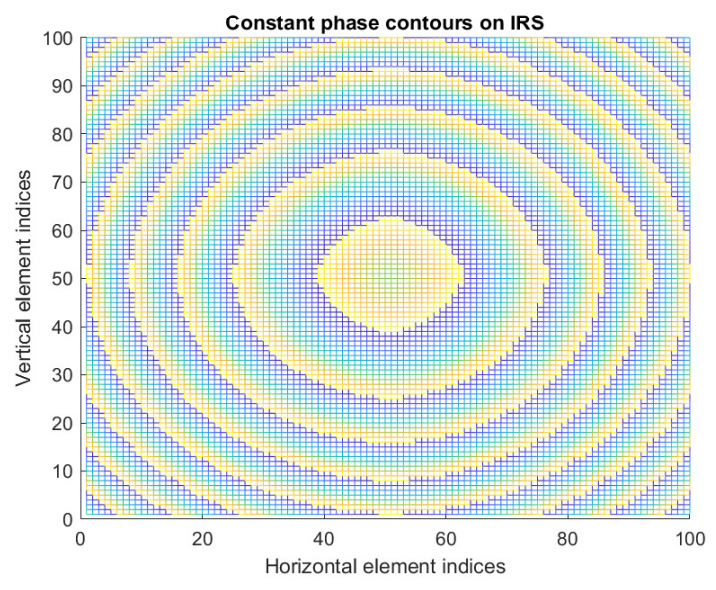

(b) UE directly in front of IRS center.

Fig. 2: Phase contours on the IRS. the phase distribution would be as illustrated in Fig. $2 b$. The near-field focusing can now be performed by subtracting the off-line calculated phase values representing the co-centric constant phase contours from the phase values found in step 1 . The correct pattern can be found with a similar search as that in step 1, i.e., the phase pattern resulting in largest received power at AP is selected to be used. The mechanical equivalent of step 2 would be changing the shape of the surface from a planar array to a parabolic reflector.

In Section IV, the searches in Steps 1 and 2 are performed with exhaustive search. However, other search methods can be used and since the environment wherein the IRS is placed is known, the search space can be limited in practical scenarios.

\section{NUMERICAL EXAMPLES}

The operation and performance of the proposed beamforming method is studied with simulations. The distance between the AP and IRS is assumed to be $10 \mathrm{~m}$ and the path loss between them is modeled with the $5 \mathrm{G}$ path loss model [10]. In addition to the path loss, the channel between the AP and IRS has a single complex Gaussian channel tap with mean power value 1 . The heights of the AP antenna and IRS center point are $2 \mathrm{~m}$. The UE antenna height is $1.5 \mathrm{~m}$. The distance and direction of the UE from the IRS is varied. The channel between the UE and IRS elements are assumed to be free space channels and IRS elements are isotropic. The noise power at the receiver in decibel milliwatts $(\mathrm{dBm})$ is calculated as $-174+10 \log _{10}(B W)+N F$, where $B W$ is the signal bandwidth and $N F$ is the noise figure of the receiver at AP. The parameters $x, y$ and $z$ defining the UE position are defined in Fig. 1 and are in all cases $x=1 \mathrm{~m}, y=-0.5 \mathrm{~m}$, $z=1 \ldots 10 \mathrm{~m}$. The distance between the IRS center point and UE is calculated as $\sqrt{x^{2}+y^{2}+z^{2}}$.

The performance of the proposed beamforming technique is measured by the loss in the received power. In the ideal case, the required phase values at the IRS elements are known perfectly. The $0 \mathrm{~dB}$ level in the figures showing the loss due to the EM field curvature in the near-field of the IRS represents the ideal case. The dashed curve (known far-field, no nearfield focusing) shows the performance when the direction from 


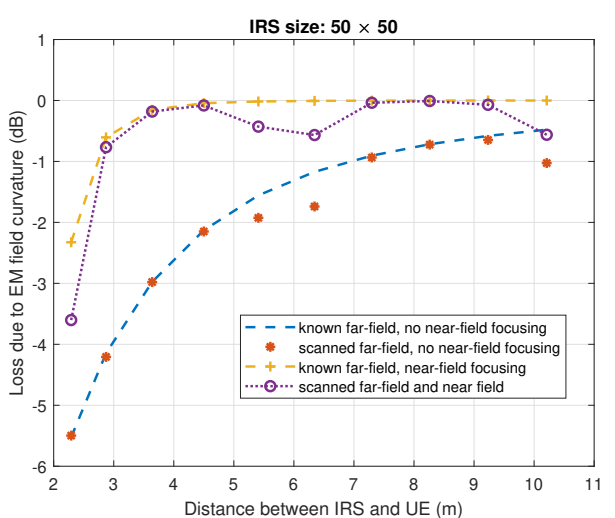

Fig. 3: Loss due to the EM field curvature in the ideal case.

the UE to the IRS is known but no attempt to perform nearfield focusing is done. The results shown with the asterisks (scanned far-field, no near-field focusing) give the performance when the direction from the IRS to the UE is found by the scanning but without near-field focusing. The dashed curve with the plus signs (known far-field, near-field focusing) gives the performance when the direction from the IRS to the UE is known and the near-field focusing is utilized. The dotted curve with circles (scanned far-field and near field) represents the cases where the direction from the IRS to the UE is first found in Step 1 of the proposed method and the near-field focusing is then performed in the step 2 .

The performance at $5 \mathrm{GHz}$ frequency with a IRS composed of $50 \times 50$ element in a noiseless case is shown in Fig. 3. The performance with the same array as that in Fig. 3 but when the transmit power at the UE is $0 \mathrm{dBm}, B W=20 \mathrm{MHz}$, and $N F=0 \mathrm{~dB}$ is shown in Fig 4. The performance with $10 \mathrm{dBm}$ transmit power at $\mathrm{UE}$ is shown in Fig. 5. The number of antenna elements in AP is two and the training sequence length is 10 symbols. The curves in Fig. 4a show the received power at the AP. The received powers are labeled as in Fig. 3 and the horizontal dashed line shows the noise power $(-101 \mathrm{dBm})$ at $20 \mathrm{MHz}$ band. As can be seen from Fig. 4a, the signal-to-noise ratio (SNR) decreases rapidly with the increasing distance between the IRS and UE. Since the received power is used to decide the phase values at IRS, the performance of the beamforming is also degrading. The difference between the cases when the near-field focusing is used with known directions and with scanned direction in step 1 indicates that the performance is is limited more by the farfield beamforming in Step 1 than by the near-field focusing in Step 2.

Fig. 6 shows the performance with an $50 \times 50$ element IRS operating at $3 \mathrm{GHz}$ with transmit power $0 \mathrm{dBm}$. The increased power at the AP in comparison with the performance at $5 \mathrm{GHz}$ is due to the increased physical size of the array. At $5 \mathrm{GHz}$ the array size $0.78 \mathrm{~m} \times 0.78 \mathrm{~m}$ is but at $3 \mathrm{GHz}$ the size is $1.3 \mathrm{~m} \times 1.3 \mathrm{~m}$.

The numerical results show that the proposed beamformer is able to compensate the power loss due to the near-field effect

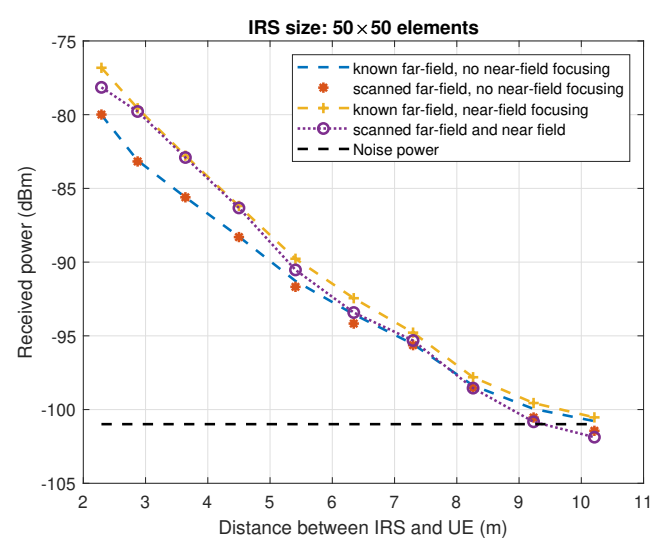

(a) Received power at AP.

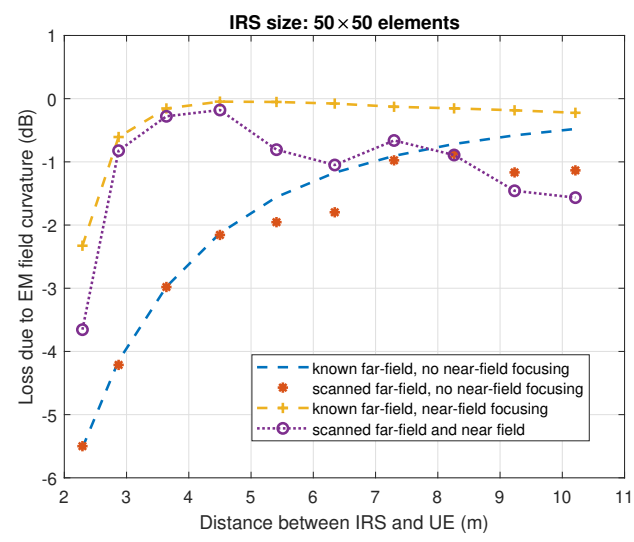

(b) Loss due to near-field effect.

Fig. 4: Performance with an IRS with size $50 \times 50$, Tx power $0 \mathrm{dBm}$.

when the UE is close to the AP. With the two-step beamformer the achieved gain in the received power at the AP is up to $3 \mathrm{~dB}$ when comparing the cases where the EM field curvature in the near-field is ignored and when it is taken into account in Step 2 of the proposed method.

\section{CONCLUSIONS}

The IRS is used to reflect the signal transmitted by an UE to an AP. Since the IRS is a passive element, it is not able to amplify the signal. Further, the UEs are typically power constrained and the size of the IRS implementation is limited, especially in indoor scenarios. Thus, the UE utilizing the IRS is likely to be relatively close to the IRS. The short distance between the UE and IRS necessitates to consider the near-field propagation, i.e., the plane wave model is not accurate enough in these cases. In this paper, a two step beamforming method is proposed. In the first step, the direction of the UE from the IRS is found assuming the plane wave model. After the direction angles have been found, the beam is focused towards the UE utilizing pre-calculated phase patterns. These patterns can be calculated and stored in memory when the size and geometry of the IRS and the operating frequency are known. The method does not require any computation, all operations can be done 


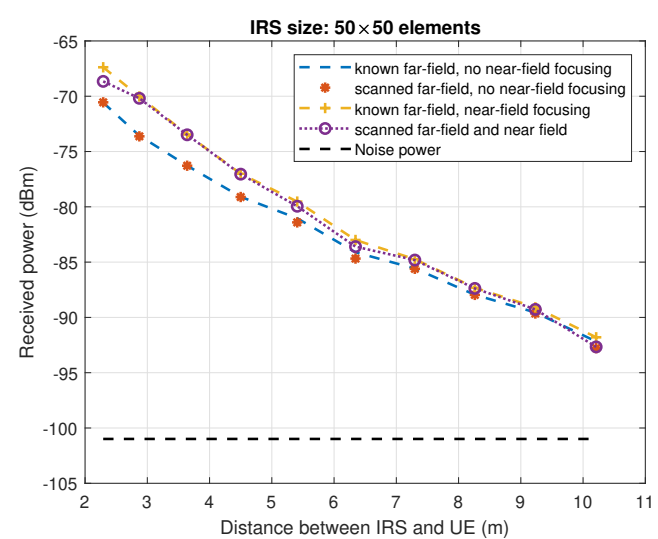

(a) Received power at AP.

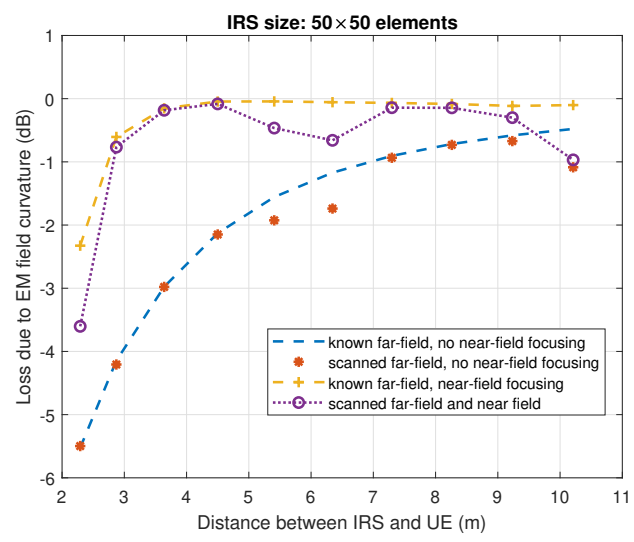

(b) Loss due to near-field effect.

Fig. 5: Performance with an IRS with size $50 \times 50$, Tx power $10 \mathrm{dBm}$.

as memory operations. The searches in this paper have been performed as exhaustive searches but simpler algorithm should be investigated in the future work. The search can be further accelerated when the information about the location of the IRS is used to narrow down the search space. The same principle can be applied also in downlink direction, but in this case a feedback channel from the UE to the IRS controller is need at the end of both steps 1 and 2 .

\section{ACKNOWLEDGMENT}

This research is partially funded by the Academy of Finland 6Genesis Flagship (grant 318927).

\section{REFERENCES}

[1] E. Basar, M. Di Renzo, J. De Rosny, M. Debbah, M. Alouini, and R. Zhang, "Wireless communications through reconfigurable intelligent surfaces," IEEE Access, vol. 7, pp. 116753-116773, 2019.

[2] M. Di Remzo, et al., "Smart radio environments empowered by reconfigurable ai meta-surfaces: an idea whose time has come," EURASIP Journal on Wireless Communications and Networking, 129(2019), 2019.

[3] Q. Wu and R. Zhang, "Towards smart and reconfigurable environment: Intelligent reflecting surface aided wireless network," IEEE Communications Magazine, vol. 58, no. 1, pp. 106-112, 2020.

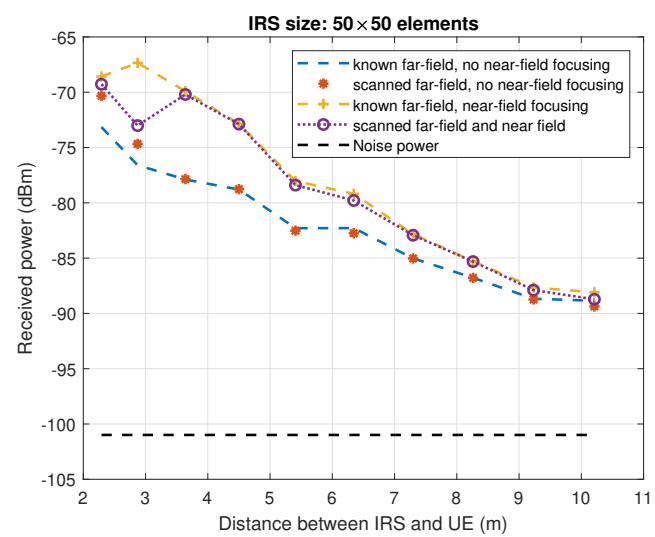

(a) Received power at AP.

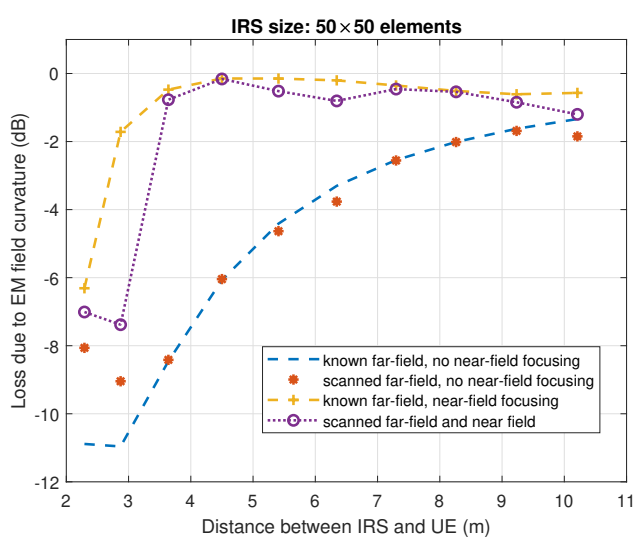

(b) Loss due to near-field effect.

Fig. 6: Performance with an IRS with size $50 \times 50,3 \mathrm{GHz}$ Tx power $0 \mathrm{dBm}$.

[4] B. Liu, A. P. Guevara, S. D. Bast, Q. Wang, and S.Pollin, "Massive mimo indoor localization with 64-antenna uniform linear array," in 2020 IEEE 91st Vehicular Technology Conference (VTC2020-Spring), 2020, pp. $1-5$.

[5] A. Dürr, B. Schneele, D. Schwarz, and C. Waldschmidt, "Range-angle coupling and near-field effects of very large arrays in mm-wave imaging radars," IEEE Transactions on Microwave Theory and Techniques, pp. 1-9, 2020.

[6] L. Yan-ping, J. Yan-min, and Z. Chao-zhu, "Robust near-field beamforming with worst case performance based on convex optimization," in Proceedings of 2012 5th Global Symposium on Millimeter-Waves, 2012, pp. 608-611.

[7] F. Zhang and W. Fan, "Near-field ultra-wideband mmwave channel characterization using successive cancellation beamspace uca algorithm," IEEE Transactions on Vehicular Technology, vol. 68, no. 8, pp. 72487259, 2019.

[8] O. Yurduseven, S. D. Assimonis, and M. Matthaiou, "Intelligent reflecting surfaces with spatial modulation: An electromagnetic perspective," IEEE Open Journal of the Communications Society, vol. 1, pp. 12561266, 2020.

[9] C. A. Balanis, Antenna Theory: Analysis and Design, 2nd edition, John Wiley Sons, Inc., 1997.

[10] "Study on channel model for frequencies from 0.5 to $100 \mathrm{GHz}$," 3GPP TR 38.901, version 14.3.0, Release 14, ETSI, 2018 